\title{
15 Years of Enterprise Architecting at HICSS: Revisiting the Critical Problems
}

\author{
Stephen H. Kaisler, D.Sc. \\ SHK \& associates \\ Laurel, MD \\ United States of America \\ Skaisler1@comcast.net
}

\author{
Frank Armour, Ph.D. \\ Kogod School of Business \\ American University, Washington, DC \\ United States of America \\ fjarmour@gmail.com
}

\begin{abstract}
The Enterprise Architecture (EA) minitrack has been a mainstay of HICSS for the past 15 years. The methodology, tools, and processes of enterprise architecting have evolved during that period. In 2005, Kaisler and Armour identified some critical challenges in modeling, management, and maintenance for EA that needed attention to ensure a viable technical discipline. Over 15 years, we have accepted 93 papers for presentation. Reviewing these papers and drawing up on our experience over the past 15 years, we conclude that some progress has been made, some challenges remain to be addressed, and some new challenges have emerged. This paper revises existing challenges and identifies additional challenges to be addressed in the next 10 years.
\end{abstract}

\section{Introduction}

The Enterprise Architecture (EA) Minitrack has resided in the Organizational Systems Track of HICSS for the past 15 years. During that time, the cochairs have observed significant changes in the perception of EA. The emergence of business architecture as an important component of the Enterprise Information technology environment has become clearer. The maintenance challenge of revising the EA - both documentation and the physical instantiation - has not been adequately addressed. Some new challenges have emerged in the areas of security, privacy, operations, and storage with the advent of newer technologies such as cloud and virtualization, and new threats to the systems from advanced persistent threats (APTs). Some progress has been made, but more work needs to be done to ensure that EA becomes a viable tool for organization's development of complex IT systems.

In 2005, Kaisler and Armour [3] presented a paper at HICSS-37 entitled Enterprise Architecting: Critical Problems based on over 20+ years of experience conducting EA activities in a number of venues. That paper identified and analyzed a set of problems that affected EA and that we believed needed to be addressed for EA to move forward as an essential component of planning and management of an organization's IT infrastructure and business operations.

Now, at the 15-year anniversary of EA at HICSS, a review of these critical problems seems appropriate to assess progress and review the state of EA research and theory as represented by the set of papers that have been submitted to and accepted by the HICSS EA minitrack.

\section{Brief Recap of Critical Problems}

In [3], Kaisler and Armour addressed three classes of critical problems that arise from political, project management, and organizational issues and weaknesses (Table 1).

Table 1. Recap of Critical Problems

\begin{tabular}{|l|l|}
\hline Problem & Description \\
\hline Modeling & $\begin{array}{l}\text { Use of formal models and/or tools to } \\
\text { describe and analyze the EA. EAs } \\
\text { must be modeled to present a clear, } \\
\text { coherent, and concise picture of the } \\
\text { baseline and target EAs and to } \\
\text { communicate this picture to the } \\
\text { stakeholders. The EA must be good }\end{array}$ \\
\hline
\end{tabular}




\begin{tabular}{|c|c|}
\hline & $\begin{array}{l}\text { enough, but does not have to be } \\
\text { perfect. Key subproblems are: } \\
\text { Business View Presence and } \\
\text { Alignment, Modeling Tool } \\
\text { Availability and Quality, } \\
\text { Stakeholders Perspectives, Handling } \\
\text { Dynamics }\end{array}$ \\
\hline Managing & $\begin{array}{l}\text { Use of practices and procedures - } \\
\text { formal or otherwise - to develop the } \\
\text { EA and manage the EA team. An EA } \\
\text { framework, such as described in [ ], } \\
\text { TOGAF, DODAF, etc., along with } \\
\text { portfolio management processes, can } \\
\text { guide the development. Key } \\
\text { subproblems are: Assessing } \\
\text { Technical Architecture Maturity, } \\
\text { Assessing Infrastructure Stress, The } \\
\text { System Architect's Value } \\
\text { Proposition, Virtual Enterprise, } \\
\text { Scalability, EA Metrics, Best } \\
\text { Practices }\end{array}$ \\
\hline Maintaining & $\begin{array}{l}\text { Use of practices and procedures - } \\
\text { formal or otherwise - to ensure } \\
\text { consistency of the EA as it evolves } \\
\text { and resolve the tension between } \\
\text { continuing operations and the } \\
\text { introduction of new or enhanced } \\
\text { services and capabilities. Key } \\
\text { subproblems include: Continuing } \\
\text { Technical Innovation, Evolving } \\
\text { Business Models, Mobility Support, } \\
\text { and Security. }\end{array}$ \\
\hline
\end{tabular}

\section{Methodology}

This is not an exhaustive survey of EA-related papers. Rather, 95 papers that directly addressed EA concepts, technology, tactics, and procedures over the past 15 years are analyzed, including the co-chair's first paper submitted to HICSS-36 in 2003. Kaisler and Armour's Critical Problems paper and this paper are excluded from this analysis as they defined (2005) and revised (2017) the challenges yielding 93 papers.

During the past 15 years, approximately 200 papers were submitted to the minitrack. Table 2 depicts the number of papers accepted to each conference (sum larger than 1.00 due to rounding). Kaisler and Armour assumed co-chairmanship in
2004. Paper submission rates have varied between 9 and 18 papers per conference year. During the past 16 years, the acceptance rate has varied between 45 and $57 \%$ with a mean of 6 papers, including several papers submitted by the co-chairs - jointly or with other co-authors. This acceptance rate has yielded two or three minitrack sessions per conference year par for the course of HICSS over those years.

Each paper was examined and classified into one of 6 categories with a catchall category of 'Other' if the paper did not seem to fit the preselected categories. The new categories are design, assessment, and governance. A paper was classified in design if it primarily focused on how to design an EA. A paper was classified in assessment if it discussed how to evaluate either the EA or the EA development process, including suggesting metrics. A paper was classified in governance if it discussed how to handle the EA governance process, including IT portfolio management. For example, Lindstrom's paper [16] in HICSS-39 discussed architectural principles, and was determined to be relevant to the governance of EA design. The other category was used (sparingly) if the paper did not seem to clearly fit into the other categories.

Table 3 presents the summary information for each of the categories. The total number of papers adds up to 101 - more than 85 because some papers were classified into two categories. An appendix listing the papers by author, conference and categories is available from the authors upon request. Readers can refer to the IEEE Digital library to retrieve the actual papers.

From table 3, it is apparent that the major focus of EA has been modeling, followed by design and managing of the EA process. The papers submitted contained a mix of theoretical approaches, case studies, and pragmatic applications of EA.

Table 2. Minitrack Papers Accepted

\begin{tabular}{|l|c|c|c|}
\hline \multicolumn{1}{|c|}{ Conference } & Year & \# Papers & \% Total \\
\hline HICSS-36 & 2003 & 1 & 1.08 \\
\hline HICSS-37 & 2004 & 3 & 3.23 \\
\hline HICSS-38 & 2005 & 8 & 8.60 \\
\hline HICSS-39 & 2006 & $5(6)^{*}$ & 6.45 \\
\hline HICSS-40 & 2007 & 6 & 6.45 \\
\hline HICSS-41 & 2008 & 5 & 5.38 \\
\hline HICSS-42 & 2009 & 6 & 6.45 \\
\hline
\end{tabular}




\begin{tabular}{|l|c|c|c|}
\hline HICSS-43 & 2010 & 6 & 6.45 \\
\hline HICSS-44 & 2011 & 8 & 8.60 \\
\hline HICSS-45 & 2012 & 6 & 6.45 \\
\hline HICSS-46 & 2013 & 7 & 7.53 \\
\hline HICSS-47 & 2014 & 9 & 9.68 \\
\hline HICSS-48 & 2015 & 9 & 9.68 \\
\hline HICSS-49 & 2016 & 6 & 6.45 \\
\hline HICSS-50 & 2017 & $8(9)^{*}$ & 9.68 \\
\hline Total & & $93(95)$ & 102.15 \\
\hline
\end{tabular}

*Kaisler and Armour's 2005 paper and this paper omitted from count.

Table 3. Paper Summary by Category

\begin{tabular}{|l|c|c|}
\hline \multicolumn{1}{|c|}{ Category } & \# Papers* & \% Category \\
\hline Design & 20 & 21.05 \\
\hline Modeling & 33 & 38.8 \\
\hline Managing & 20 & 21.05 \\
\hline Maintaining & 4 & 4.21 \\
\hline Assessment & 8 & 8.42 \\
\hline Governance & 9 & 10.53 \\
\hline Other & 10 & 13.68 \\
\hline
\end{tabular}

* Some papers appear in two categories.

\section{EA Challenges: The Next 10 Years}

Based on the analysis and review of these 93 papers, we believe that over the next 10 years enterprise architecting will continue to face the same challenges first identified in 2005 as well as new challenges - both of which are discussed in the following sections.

4.1 EA Design Tools. In [3], it was noted there was a dearth of good modeling tools that would aid an EA team in developing requirements representing the as-is and to-be architectures, tracking EA progress, and assessing the EA from a quality attributes perspective.

A separate category, EA Design, has been introduced to bring greater awareness to the differences and some of the challenges associated with EA design. We believe there are three core elements required for modeling and design tools: (1) a consistent representation scheme using a number of different views to capture different aspects of the architecture; (2) a consistent naming and referencing mechanism, supported by a data dictionary, across all views; and (3) a shared EA repository to store, retrieve, and cross-reference these EA artifacts.
Given these three elements, different tools may be used for different views and different functions as long as they use the repository which provides a set of canonical views.

Since 2005, both changes and some progress has been made in EA design and modelling tools. Multiple tools have disappeared and there has been consolidation through acquisition and merger. But, end-to-end analysis, design, test, implementation, and visualization tools with representation and assessment of quality attributes and metrics in a single tool have not yet matured to fully support EA processes. The website, www.modaf.com lists a number of tools that the reader might want to consider (we are not recommending any of these, just providing a reference). Table 4 addresses some of the design challenges that have arisen since the original paper.

Table 4. EA Design Challenges Challenge

Security \& Privacy: Multiple security breaches over the past decade, such as the Target breach in December 2013 that led to exposure of customer's credit card information, continue to demonstrate the need for security and privacy mechanisms and policies as key elements. Within EA, security and privacy mechanisms and policies need to be designed into every aspect of the architecture as opposed to relying on the underlying systems software to provide these capabilities.

Moving Beyond the Cloud: A decade ago EAs were focused on service-oriented architecture (SOA) implementations. Recently, enterprise architects have begun to embrace cloud computing-based solutions, which introduce another level of complexity into EA design. Few design/modeling tools accommodate cloud computing approaches. The paradigm beyond the cloud is not yet clear, but EAs will most likely be forced to transition to it as it gains acceptance. Kaisler, Money and Cohen [10] describe a decision framework for cloud computing adoption.

Open Source Software: Although many organizations have adopted open source software (OSS), it remains a challenge to use because organizations must work around the features that do not support their needs to conform OSS to their business operations. Lack of documentation in OSS is a persistent and ongoing problem which stifles effective use. Moreover, because OSS is being decomposed into more explicit layers of software, there are often significant integration challenges in making OSS interoperable.

Big Data: Designing for Big Data presents major 
challenges when one considers storing, organizing, moving, analyzing and visualizing large amounts of data on the order of petabytes. Kaisler, Armour, Espinosa and Money [11, 12] identified some of the issues and challenges of Big Data and how to obtain value using big data in the context of on-line service delivery [13].

New technology Stresses: With new technologies, such as affordable and active smart sensors, a flexible architecture is required that responds to events that are exceptions rather than the operational norm. Commoditization continues to drive computing hardware prices downward. Innovation resides mostly in software systems, but these often have short lifetimes with constant pressure to keep up with the "newest and greatest".

Microservices: A microservice is a small application configured as a set of small services. Each application runs in its own process. Each application communicates with lightweight mechanisms, often using an HTTP resource API, such as REST. Microservices are revolutionizing the design of web-based applications across many domains. By decomposing functionality to a granular level, reusability of low-level functionality is increased. But, the tradeoff is often the performance hit taken as a result of the communication mechanisms.

Armour, Kaisler and Liu [1] viewed security as orthogonal to the structure of an EA, e.g., a persistent design requirement that needs to be integrated into the EA from the Business View through to Technology View. The authors included Privacy within this set of requirements. It is now clear that Security and Privacy are two different design requirements in EA although there is strong interaction between them. For example, Privacy imposes more stringent requirements and legal liabilities - criminal, civil, and financial - than Security, such as violations of the Health Insurance Portability and Accountability Act in the United States.

Additional artifacts are required in an EA: identification of security and privacy vulnerabilities, defensive technologies, and mitigating practices to ensure security and privacy compliance with appropriate regulations, among others. There is a strong emphasis on a security architecture that is both embedded in the EA and co-located with each component of an EA. But, no papers addressed the co-development of a security architecture as an essential element of the EA.
4.2 EA Methodology. A methodology specifies how enterprise architecting is to be performed to yield an EA. Many frameworks do not include a methodology, although the TOGAF, DODAF, and the TISAF [1, 2] did so. The DODAF [6] is mandated by the U.S. Department of Defense for most of its enterprise architecture efforts. The TOGAF [23] has been widely accepted by commercial firms in the U.S. and Europe. Within a methodology, there are numerous challenges such as skill sets, using agile practices, and training for team members that must be addressed. A few recent paper have addressed applying agile methods to the enterprise architecting process.

Two architecture maturity models have been developed to assess EA methodology results: the NASCIO's Enterprise Architecture Maturity Model (EAMM) [9] and the Architecture Capability Maturity Model (ACMM) [17]. The EAMM followed the structure of the Software Engineering Institute's Capability Maturity Model (CMM). It was released in 2003, but has not been significantly updated since.

The U.S. Department of Commerce's ACMM, which was revised in 2007, was developed to assist agencies in assessing their progress towards repeatable EA processes. It contains six levels and nine architectural elements. It is noteworthy in that it specifies two methods to calculate a maturity rating. The first method obtains a weighted mean IT architecture maturity level. The second method shows the percent achieved at each maturity level for the nine architecture characteristics.

The lack of modern maturity models given the progress in software engineering methodologies remains a significant challenge in assessing the success and value of EA processes. A modern maturity model will have to address agile methodologies as an element of the EA processes.

Table 5 addresses some of the current methodology challenges that we have identified in our reviews.

\section{Table 5. EA Methodology Challenges}

\begin{tabular}{|l|}
\hline \multicolumn{2}{|c|}{ Challenge } \\
\hline Credibility: Well into its third decade, EA still faces a \\
credibility challenge as many business operations \\
managers do not see the value returned for the \\
investment made. Associated challenges are managerial \\
change resistance and low management priority. As we \\
\hline
\end{tabular}




\begin{abstract}
noted, credibility must flow from the top down in an organization. While some papers identified senior executive support, most case studies focused on middle management below the Cxx level. We believe that few senior executives have the understanding of EA to convey this message to lower levels of the organization. Better communication from mid-level managers to executives is required to impart this understanding of how EA can benefit the organization.

Compliance: Compliance with standards and regulations was only briefly cited in early frameworks, [1] for example. The emergence of compliance as a major management challenge, also makes it a major methodology challenge as each stage (see [2] for example) must incorporate methodological practices to ensure that compliance with standards and regulations are satisfied. A separate issue is a methodology for the assessment and enforcement of an IT project's compliance with the EA. Some evidence for compliance assessment was found in the HICSS papers, but it may be or is more likely to be covered in IT project methodologies. Because of the legal and financial implications of compliance, it must be a pervasive process within both EA management, EA design, and EA assessment methodologies.

Repeatability: Each application of an EA methodology is usually customized to adapt to a particular customer's requirements. Thus, a comparison of the repeated application of an EA methodology to different organizations is hard to come by. None of the HICSS papers have focused on repeatability, although many claim the EA methodology is designed for it.
\end{abstract}

4.3 EA Modeling. Modeling methodology and tools was the largest category of papers. However, this fact is mitigated by the variety of models and modeling approaches and tools used in the various papers. Only a few commercial tools, such as Rational's products, were cited; many modeling tools resulted from academic research for advanced degrees. EA models were also varied - from requirements and conceptual design through implementation to the management and assessment of the architecting process and its associated design artifacts. Table 6 identifies several near-term challenges.

Several open source modeling tools have been developed, including the TOGAF Customiser (http://www.opengroup.org/togaf/epf_intro.html), ArchiMate (http://archi.cetis.ac.uk/) and Modelio (http://www.modeliosoft.com/). Several EA modeling languages have been proposed, but lacking a standard vocabulary and set of architectural constructs, it is difficult to compare them. In the nearterm, new head-to-head comparisons of EA modeling tools and languages using a set of well-defined features are needed to help architects select the most appropriate tool and language for their EA effort.

Open issues include: What are the basic components of an EA that should be modeled? And, to what granularity? For example, we believe that an EA model must also consider the locations of business operations and the constraints they place on an EA. Zachmann [24] and our EA framework [1, 2] are two of a few EA frameworks that consider these EA elements. Le and Wegmann [14] suggest that a modeling language should provide multiple levels with consistent principles across the levels; the ability to model actions between systems and levels both spatially and temporally; and traceability of the relations between systems across different levels.

Herbert Simon [22] noted, "Modeling is a principal -perhaps the primary - tool for studying the behavior of large complex systems". In this era of globalization, complexity across national and international boundaries is inevitable. An equally critical issue is how much support is provided for modern IT and software engineering approaches such as cloud computing, service science, agent-oriented and security reference architectures, among others.

According to the Second Law of Software Evolution, the complexity of a software system will increase if no explicit action is taken to avoid it [Lehman]. Complexity can lead to increased costs, possible lack of understanding of an EA's functionality, and lack of agility in responding to business environment changes. Functional complexity may lead to problems in terms of operation stability, reliability, integration, response time, cost etc. But, complexity is a fuzzy term and has many stakeholder perspectives. Reconciling those perspectives depends on a standard vocabulary and a standard set of metrics.

Table 6. EA Modeling Challenges Challenge

Standard EA Ontology and Vocabulary: A standard ontology to develop a formal model of an EA and, thus, be the basis for different modeling tools is required. An ontology is the working model of entities and interactions in some particular domain of knowledge or 
practices. The ontology must address (1) what are the basic components of an EA that should be modeled, and (2) at what level of granularity with what features should these components be described. Concurrently, a standard vocabulary is needed for EA modeling to enable comparison among different models.

Modeling Quality Attributes: Modeling systems, including languages, are required to facilitate assessment of EAs according to quality attributes.

Effective communication between the various EA stakeholders: An EA specification contains multiple viewpoints, including the technical, data and business views. With multiple stakeholders, with their expertise and "language", it is a continuing challenge to specify and communicate an EA specification that provides a common representation that can be understood by all stakeholders, a strong common ground, so to speak. Research that has been presented at the minitrack on team knowledge in enterprise architecting [7] (Espinosa 2013) supported the use of the Data Architecture as the one view that all stakeholders can understand and therefore utilize as means of common understanding.

The Devil in the Details: EA modeling needs to go beyond just nodes - whether hardware, software or both - to information exchange protocols - whether network or interprocess. A richer syntax is required to specify or describe quality attributes.

Agile Enterprise Architecture specification and deployment: A key criticism of early EA initiatives was that the overall effort was huge, unwieldy and time consuming. The overall effort to complete the various views of an EA framework at an enterprise level can involve a long timeframe and large teams, with the end result already being out of alignment with ever changing business needs. Recently, the minitrack has seen multiple papers that discuss a more agile approach to Enterprise architecture that include light documentation and iterative approaches with faster cycle times to ensure more ongoing business stakeholder involvement. However, this issue remains a key challenge, in which additional research is needed.

4.4 EA Management and Governance. Every organization should have as a goal to develop a welldefined, disciplined, managed, and mature EA process as this can contribute to productivity and success. In [3], we indicated that governance and IT portfolio management were emerging concerns in EA process management. One of us (Armour) has been intensely involved in IT Governance and IT Portfolio Management process development.
Architecture governance [19] is the process of managing the design and development of the EA through its life cycle. It must be closely linked to IT Governance, which is the process of aligning IT strategy with business strategy to achieve business goals and measure IT performance and IT support for business operations. It is a maturing discipline that, properly executed, can help attain success in enterprise architecting.

Closely associated with IT Governance is IT Portfolio Management which manages the set of IT assets - hardware, software, and networks - deployed by an organization to support its business operations. But, portfolio management is more than that - it is the management of utilization, modernization, and scheduling of business and IT assets [19]. Table 7 presents some of the management challenges.

Table 7. EA Management Challenges

\begin{tabular}{l} 
Challenge \\
\hline Compliance: Since 2005, business systems must be in \\
compliance with a number of Federal laws/ regulations, \\
such as Sarbanes-Oxley, and standards, such as CoBIT. \\
Many countries are introducing new compliance regimes \\
because of the world-wide depression/recession. A key \\
question for enterprise architects not addressed in these \\
papers is how to evolve the EA, including introducing \\
new technology and processes, while continuing to \\
maintain compliance with regulatory mechanisms and \\
standards.
\end{tabular}

Integration: Most EAs integrate multiple business systems into a coherent picture for the organization. It is sometimes a technical challenge, but (almost) always a management challenge. We observe that there is no well-developed methodology for integrative design and management.

Capability Maturity Assessments: Every organization should periodically perform a capability maturity assessment of its EA processes to determine if improvements are needed. EA capability maturity is not a static state, but a continually evolving process as the technology evolves and the business environment changes.

Sponsorship: Many of the case studies described the participants in workshops and interviews, but only a few of these had C-level participation. Lack of sponsorship at the C-level (e.g., CIO, CFO, etc.) continues to lead to lack of full success rather than absolute failure. Organizations that have only partial success often cannot reap the benefits of an enterprise-wide architecture.

Lack of alignment with Business Strategy: In the past 


\begin{abstract}
many early EA initiatives tended to be driven from the technology perspective and lacked a strong alignment with business goals, objectives and processes. The challenge is not only to be better able to align with the business needs, but engage business stakeholders. In the past several years the EA minitrack has seen an increasing number of research results that focus on a business architecture driving the EA. Managing EA initiatives in this way gives the business stakeholders ownership of the EA, but it will continue to be a challenge.

Security: Creating pliant systems that lead to flexible business functionality often creates security challenges.

The threats are constantly shifting and evolving. Proactive security management requires constant hardening of an organization's systems.
\end{abstract}

4.5 EA Maintenance. Based on the four submissions to HICSS, very little effort is being devoted to issues and tools for maintaining an EA after its initial implementation - from an academic perspective. And yet, as indicated in [2], considerable effort must be dedicated to both maintaining the existing EA as well as enhancing it to satisfy the requirements for the next iteration.

As mentioned in [2], EA maintenance is encompassed by the four Rs: Replace, Revise, Refresh, and Retire. Each has cost, schedule and functionality implications. As Rechtin [18] observed, you can have two of the three, but not all three.

A critical aspect of EA maintenance is ensuring that the EA model is synchronized with the real world. Because IT architecture maintenance projects often have different schedules from development projects, continual vigilance is required to ensure that the EA reflects the current physical IT architecture. Thus, in today's dynamic environment, EA processes must incorporate adaptive processes for updating to reflect EA maintenance activities. We have identified only a few papers within the literature that address this problem. Table 8 presents some of the maintenance challenges.

Table 8. EA Maintenance Challenges

\begin{tabular}{|l|}
\hline Challenge \\
\hline Technology Refreshment: With HW/SW technology \\
evolving rapidly, new technology must be integrated \\
into the EA and old technology retired from the EA \\
without disrupting current operations. The refresh cycle \\
is decreasing and the apparent turnover in technology \\
\hline
\end{tabular}

seems to be accelerating with a reduced lifetime of utility of many software development and support tools. Impact of Rapid Environmental Change: A critical issue is how to handle steady, dynamic change especially when many concurrent IT projects are being developed? How does one keep the EA artifacts synchronized with the project's evolutions?

4.6 EA Assessment. EA assessment encompasses two key areas: quality attributes and metrics. These subareas encompass both evaluation and measurement of methodological practice with its associated artifacts and the resulting architecture.

Quality attributes assess the tangible and nontangible properties of the EA, but are hard to define. One such attribute is value, which has multiple levels. One can consider cost avoidance and cost reduction as two elements. But, so is risk reduction? And, stakeholder value? And, increases in productivity? Each of these has different units of valuation, but must be resolved to a canonical concept of value to yield a useful and actionable measure for an organization's executives.

Applying metrics to EA has two connotations. First, defining and implementing metrics about the EA process, which was addressed above. Second, defining and applying metrics to the EA artifacts and implementation processes. According to Schulz et al. [20], structural system complexity is related to number and heterogeneity of elements and relationships as $\mathrm{C}_{\mathrm{EA}}=\left(\mathrm{N}_{\mathrm{E}}, \mathrm{N}_{\mathrm{R}}, \mathrm{H}_{\mathrm{E}}, \mathrm{H}_{\mathrm{R}}\right)$. From this, they compute an entropy measure similar to that used by Claude Shannon in his theory of communications. This seems to represent a first cut at a complexity metric, but remains to be validated. The open question is: Are other metrics needs? And, what are they?

Table 9 presents some critical EA Assessment challenges.

Table 9. EA Assessment Challenges

\begin{tabular}{|l|}
\hline Challenge \\
\hline Standard EA Metrics: Outputs to Outcomes: Many EA \\
teams produce numerous EA artifacts, but few have \\
metrics that are routinely measured and reported. We \\
termed this the "outputs to outcomes" problem because \\
there is often no direct linkage between the quantity of \\
EA artifacts and the quality of the EA itself, if it has \\
been measured at all. GAO [9] identified this as a key \\
problem in its report on organizational transformation. \\
\hline
\end{tabular}




\begin{tabular}{l} 
There has been no method for evaluating the quality of \\
EA artifacts. The complexity and diversity of EA \\
artifacts makes it difficult to develop a common \\
evaluation method. \\
\hline The Need for KPIs: The Key Process Indicator (KPI) \\
concept is a useful one, but general KPIs may not work \\
for every organization because of its culture and politics. \\
When an organization chooses an EA methodology, it \\
should also develop its KPIs. KPIs need to be both \\
quantitative and qualitative. Guidance for developing \\
EA KPIs is required. \\
\hline Measuring EA Value: Value is hard to quantify and \\
qualify because it has many levels. On one level, \\
executives often ask how EA can or will contribute to \\
their business operations. On another level, executives \\
often ask how EA can or will ensure that their IT \\
infrastructure and applications can respond in an agile \\
manner to a changing and rapidly evolving marketplace. \\
\hline
\end{tabular}

4.7 EA Frameworks. Several EA frameworks have been developed and applied to projects of various sizes, including Zachmann [24], TOGAF [23], FEAF [8], and DODAF [6]. No one framework has been deemed superior to any of the others. Moreover, it is not clear that any one framework will fit all EA needs. DODAF is mandated by the U.S. Department of Defense for many DOD IT-based systems. Many industrial, commercial, governmental, and academic organizations have adopted some variation of Zachmann or TOGAF.

A critical challenge that persists is a good theory and scientific foundation for EA development and methodology. This lack limits our ability to compare EA frameworks, EA artifacts, and the enterprise architects themselves.

Table 10 addresses some of the challenges in developing new frameworks or extending existing frameworks to encompass some of the challenges described in the previous sections.

Table 10. EA Framework Challenges

\begin{tabular}{|l|}
\hline Challenge \\
\hline Essential Artifacts: The frameworks mentioned above \\
(and others) have a varied array of artifacts that \\
constitute the requirements for describing an EA. There \\
is overlap among these frameworks, perhaps as much as \\
$70 \%$ in some cases. A major research question is what \\
constitutes the set of essential artifacts (the minimum \\
set) to appropriately describe an EA? Alternatively, is \\
there a common foundation for an EA framework?
\end{tabular}

EA Framework Extensions: As EAs have become more complex, greater attention has to be paid to the IT assets that comprise the EA. In particular, extending the EA framework to encompass an information system architecture (ISA) focused on the business processes and a software system architecture (SSA) focused on the technical details of the software seem to be required.

No Silver Bullets: There are over 90 or so EA frameworks extent in the technical literature or described on the web. All of them have failed at least once, and many of them have failed many times over. There are a few major ones: Zachmann, TOGAF, DODAF, FEAF, etc. When choosing an EA framework, we recommend that you read the majors and some of the minors. Decide which framework best fits your organization's modus operandi rather than just picking one of the majors. Some of the majors require substantial effort. An organization may be better served by a minor framework that is less onerous, less labor-intensive to implement.

4.8 Other Challenges. As noted above, the Other category identified some aspects of EA and the EA process which did not fit the other categories. Across the 15 years only one or two papers in each of the subtopic areas were accepted. In future efforts, we will explore these other categories in more detail to determine their overall impact on enterprise architecture and the process of architecting.

\section{Conclusions}

While progress has been made in addressing the critical problems identified in [3], it has been neither consistent nor yielded a definitive set of repeatable and measurable methodologies, principles, and practices. This paper has assessed progress as reflected in the HICSS submissions and found that in multiple areas progress has been uneven. While this paper considered only the papers submitted to HICSS, the co-chairs have also served as co-chairs and reviewers for the EA minitrack for the American Conference on Information Systems (AMCIS-16 through AMCIS-23), which has provided additional perspective on EA activities. Additionally, new areas have been identified and addressed as a result of our further investigations and analysis of the HICSS papers. 
So, what do we conclude from our retrospective review of the HICSS papers? The following observations and critical challenges have been distilled from experience.

1. Despite the large amount of research in EA, the EA community has yet to resolve some of the critical problems that affect adoption of EA. Among these are lack of standard vocabulary, lack of an ontology, lack of end-to-end modeling and design tools, lack of a standard modeling/description language, and lack of metrics.

2. Business organizations large and small do not yet fully understand the value of enterprise architecture and enterprise architecting. The concepts of Return on Investment (ROI)) and Total Cost of Ownership (TCO) have been discussed in a few papers, but there is no formal model nor set of metrics for valuing the contributions of EA to the bottom line.

3. The pace of technology innovation is accelerating, and continues to challenge IT managers with new technologies, approaches and risks. Consider the recent examples of agile development, cloud computing, mobile technology, serviceoriented design and delivery, microservices, open source software, and further commoditization of hardware and software. Many businesses, seeing the light, are outsourcing IT with positive effects on their bottom line. These technologies and the decision to outsource IT services place significant strain on developing enterprise architectures and synchronizing them with business operations. The need for flexible, extendable, and robust enterprise architectures that can adapt to changing business conditions and accept or integrate new technologies well is clearly indicated.

4. Many studies and literature all show that security and privacy are critical and mandatory at many layers of IT architecture and business architecture. The architectural impact of these requirements and constraints upon performance, agility, access, and bottom line are not always positive. We believe there is a need for EAs of the future to allocate more resources to these areas, and that the architects be more creative in developing protective schemes that address these issues.

5. We believe that head-to-head comparisons of modeling tools will be beneficial in helping architects select the tool most appropriate to their EA effort.
We encourage more studies of this nature, although we recognize one tool will not fit all situations.

6. We suggest that an Enterprise Architecture manifesto should be developed that will help to focus research, development, and practice in EA just as the Agile Manifesto seemed to do 15 years ago. This manifesto should include an emphasis on up-to-date maturity models for assessing both enterprise architectures and the EA methodology.

7. There is a lack of tools for visualizing both prospective and actual enterprise architectures - both at high levels and descending to lower levels. Research is required to best convey not only structure but also features and flows of data and control within an EA.

Leaping 10 to 15 years ahead, we believe we will still be talking about some or all of EA problems and challenges elucidated in this paper.

We are continuing to examine how to improve Enterprise Architecture processes and methodologies as part of our research efforts. To this end, we will perform an in-depth analysis of the accepted papers by category to determine whether gaps are occurring in the category. This analysis may be augmented by external papers from other conferences or journals. We hope to report on this analysis at HICSS-51. Future papers by some of our research group will explore specific issues and challenges raised in this paper. In particular, we intend to address EA governance and EA security architecture and principles.

Acknowledgement: The Authors would like to thank the reviewers for their comments which have helped to improve this paper. Dr. William Money provided additional insights to several aspects of this paper.

\section{References}

[1] Armour, F., Kaisler, S., and S. Liu. "A Big Picture Look at Enterprise Architectures", IEEE IT Professional, Vol. 1(1), 1999.

[2] Armour, F., Kaisler, S., and S. Liu. "Building an Enterprise Architecture Step-by-Step", IEEE IT Professional, 1(3), 1999 
[3] Kaisler, S. and F. Armour. 2005. "Enterprise Architecting: Critical Problems", $38^{\text {th }}$ Hawaii International Conference on System Sciences, Waikoloa, HI

[4] Armour, F., Emery, C., Houk, J., Kaisler, S., and Kirk, S., "The Integrated Enterprise Life Cycle: Enterprise Architecture, Investment Management and System Development", Proceedings of the 40th Annual Hawaii International Conference on System Sciences (HICSS 2007), January 2007

[5] Dept. of commerce (DOC). 2007. Architecture Capability Maturity Model, Version 1.2, Washington, http://ocio.os.doc.gov/ITPolicyandPrograms/Enterprise_Ar chitecture/PROD01_004935

[6] Dept. of Defense (DOD). 2013. The DoDAF Architecture Framework, version 2.02, http://dodcio.defense.gov/Library/DoDArchitectureFra mework.aspx

[7] Espinosa, J. A., F. Armour, W. F. Boh, and M. A. Clark. 2013. Team Knowledge in Enterprise Architecting, Proceedings of the 46th Annual Hawaii International Conference on System Sciences, Wailoa, Maui, HI

[8] Executive Office of the President. 2007. FEA Consolidated Reference Model Document, Version 2.3

[9] Government Accountability Office (GAO). 2012. Organization Transformation: Enterprise Architecture Value Needs to be Measured and Reported, Washington, DC, GAO-12-791

[10] Kaisler, S., W. Money, and S. Cohen. 2012. "Cloud Computing: A Decision Framework for Small Businesses", published in Cloud Computing Service and Deployment Models, edited by A.M. Bento and A.K. Aggarwal, IGI Global, Hershey, PA (Chapter 8)

[11] Kaisler, S., F. Armour, A. Espinosa, and W. Money. 2013. "Big Data: Issues and Challenges Moving Forward", $46^{\text {th }}$ Hawaii International Conference on System Sciences, Grand Wailea, Maui, HI

[12] Kaisler, S., F. Armour, W. Money, and A. Espinosa. 2014. "Big Data: Issues and Challenges", Encyclopedia of Science and Technology, $3^{\text {rd }}$ Edition, IGI Global, New York, NY

[13] Kaisler, S., F. Armour, W. Money, and A. Espinosa. 2016. Obtaining Value through Big Data for Service Delivery, Business Expert Press, Chicago, IL.
[14] Le, L-S. and A. Wegmann. 2005. "Definition of an Object-Oriented Modeling Language for Enterprise Architecture", $38^{\text {th }}$ Hawai'i International Conference on Systems Sciences, Waikoloa, HI

[15] Lehman, M. M. 1996. "Laws of Software Evolution Revisited", in Montangero, C, Eds. Software Process Technology - Proceedings of the $5^{\text {th }}$ European Workshop, Spring Verlag, Berlin, pp. 108-124.

[16] Lindstrom, A. 2006. "On the Syntax and Semantics of Architectural Principles", 39 $9^{\text {th }}$ Hawaii International Conference on System Sciences, Poipu, HI

[17] National Association of State CIOs (NASCIO). 2003. Enterprise architecture Maturity Model, 1.3, http://www.nascio.org/publications/documents/nascioeamm.pdf.

[18] Rechtin, E. 1996. The Art of System Architecting, CRC Press

[19] Schwartz, K. 2007. What is IT Governance? http://www.cio.com/article/111700/IT_Governance_Definit ion_and_Solutions\#what

[20] Schulz, A., T. Widjaja, and J. Kaiser. 2013. "Complexity in Enterprise Architectures Conceptualization and Introduction of a Measure from a System Theoretic Perspective", European Conference on Information Systems (ECIS)

[21] Scott, J. 2010. A Multitude of Challenges for Business Architects, Forrester Research, http://blogs.forrester.com/jeff_scott/10-08-09$\underline{\text { multitude_challenges_business_architects }}$

[22] Simon, H.A. 1990. "Prediction and prescription in systems modeling”. Operations Research, 38(1):7-14.

[23] The Open Group. 2013. TOGAF Version 9.1 Enterprise Edition, https://www.opengroup.org/togaf/

[24] Zachman, J.A. 1987. "A framework for information systems architecture". IBM Systems Journal 26(3): 276-292 